
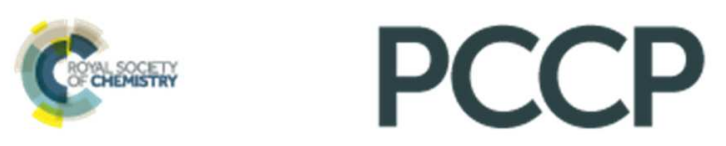

\title{
Visualisation of Quantum Evolution in the Stern-Gerlach and Rabi Experiments
}

\begin{tabular}{|r|l|}
\hline Journal: & Physical Chemistry Chemical Physics \\
\hline Manuscript ID: & CP-ART-12-2014-005606 \\
\hline Article Type: & Paper \\
\hline Date Submitted by the Author: & O2-Dec-2014 \\
\hline Complete List of Authors: & $\begin{array}{l}\text { Utz, Marcel; University of Southampton, School of Chemistry } \\
\text { Levitt, Malcolm; University of Southampton, School of Chemistry } \\
\text { Cooper, Nathan; University of Southampton, School of Physics } \\
\text { Ulbricht, Hendrik; University of Southampton, School of Physics }\end{array}$ \\
\hline
\end{tabular}




\section{PCCP Guidelines for Referees}

\section{Physical Chemistry Chemical Physics (PCCP) is a high quality journal with a large international readership from many communities}

Only very important, insightful and high-quality work should be recommended for publication in PCCP.

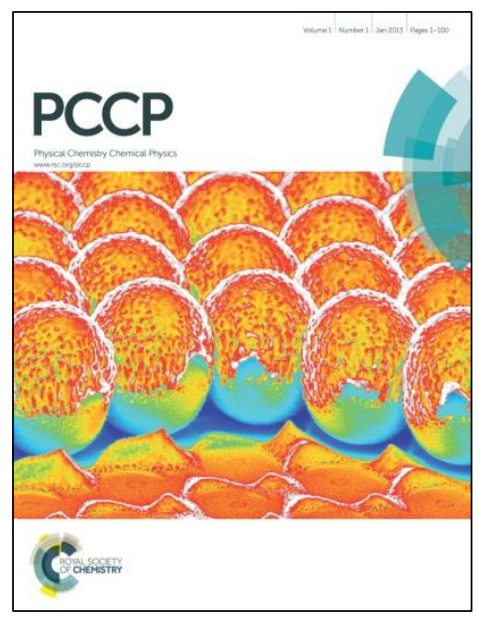

To be accepted in PCCP - a manuscript must report:

- Very high quality, reproducible new work

- Important new physical insights of significant general interest

- A novel, stand-alone contribution

Routine or incremental work should not be recommended for publication. Purely synthetic work is not suitable for PCCP

If you rate the article as 'routine' yet recommend acceptance, please give specific reasons in your report.

Less than $50 \%$ of articles sent for peer review are recommended for publication in PCCP. The current PCCP Impact Factor is $\mathbf{4 . 2 0}$.

$P C C P$ is proud to be a leading journal. We thank you very much for your help in evaluating this manuscript. Your advice as a referee is greatly appreciated.

With our best wishes,

Anna Simpson (pccp@rsc.org)

Prof Daniella Goldfarb

Managing Editor, PCCP

Chair, PCCP Editorial Board

\section{General Guidance (For further details, see the RSC's Refereeing Procedure and Policy)}

Referees have the responsibility to treat the manuscript as confidential. Please be aware of our Ethical Guidelines which contain full information on the responsibilities of referees and authors.

When preparing your report, please:

- Comment on the originality, importance, impact and scientific reliability of the work;

- State clearly whether you would like to see the paper accepted or rejected and give detailed comments (with references) that will both help the Editor to make a decision on the paper and the authors to improve it;

Please inform the Editor if:

- There is a conflict of interest;

- There is a significant part of the work which you cannot referee with confidence;

- If the work, or a significant part of the work, has previously been published, including online publication, or if the work represents part of an unduly fragmented investigation.

When submitting your report, please:

- Provide your report rapidly and within the specified deadline, or inform the Editor immediately if you cannot do so.

We welcome suggestions of alternative referees. 


\title{
Visualisation of Quantum Evolution in the Stern-Gerlach and Rabi Experiments
}

\author{
Marcel Utz, ${ }^{* a}$ Malcolm H. Levitt, ${ }^{a}$ Nathan Cooper, ${ }^{b}$ and Hendrik Ulbricht ${ }^{b}$
}

\author{
Received Xth $X X X X X X X X X X 20 X X$, Accepted Xth $X X X X X X X X X 20 X X$ \\ First published on the web Xth $X X X X X X X X X X 200 X$ \\ DOI: 10.1039/b000000x
}

The Stern- Gerlach experiment is a seminal experiment in quantum physics, involving the interaction between a particle with spin and an applied magnetic field gradient. A recent article [Wennerström et al., Phys. Chem. Chem. Phys., 2012, 14, 1677-1684] claimed that a full understanding of the Stern-Gerlach experiment can only be attained if transverse spin relaxation is taken into account, generated by fluctuating magnetic fields originating in the magnetic materials which generate the field gradient. This interpretation is contrary to the standard quantum description of the Stern-Gerlach experiment, which requires no dissipative effects. We present simulations of conventional quantum dynamics in the Stern-Gerlach experiment, using extended Wigner functions to describe the propagation of the quantum state in space and time. No relaxation effects are required to reproduce the qualitative experimental behaviour. We also present simulations of quantum dynamics in the Rabi experiment, in which an applied radiofrequency field induces spin transitions in the particle wave.

\section{The Stern-GerlachExperiment}

It would be hard to exaggerate the influence of the 1922 SternGerlach (SG) experiment on modern physics. The splitting of the beam of silver atoms into two branches by an applied magnetic field gradient is a stunningly lucid demonstration of the two quantized angular momentum states of an unpaired electron with spin-1/2, as well as the link between angular momentum and magnetism. Although the actual Stern-Gerlach experiment, as originally performed, includes elements of serendipity ${ }^{1}$ and is not quite as straightforward as depicted in standard textbooks, the experiment stands out as a milestone in quantum theory and atomic physics.

It is therefore surprising that the quantum mechanism of the SG experiment remains contentious. It has even been claimed that quantum mechanics might not be involved at all ${ }^{2}$. A recent paper by Wennerström and Westlund $(\mathrm{WW})^{3}$ studies the SG experiment by using dynamical simulations of particle trajectories and concludes that in some circumstances, dephasing of the electron spin by an external agency is necessary to explain the results. The dephasing is attributed to interactions of the traversing particle with the electron spins in the magnetic pole pieces that generate the magnetic field gradient. This interpretation runs contrary to the standard treatment of the SG experiment, which contains no elements other than the spin$1 / 2$ particle and the applied magnetic field gradient, which is considered to be time-independent and free from fluctuations.

The standard quantum theory of the SG experiment involves quantum states possessing both space and spin degrees of freedom. For the sake of concreteness, consider the case in which the particle beam moves in the $z$-direction, while the magnetic

\footnotetext{
a School of Chemistry, University of Southampton, United Kingdom SO17 1BJ.

${ }^{b}$ School of Physics and Astronomy, University of Southampton, United Kingdom SO17 1BJ.
}

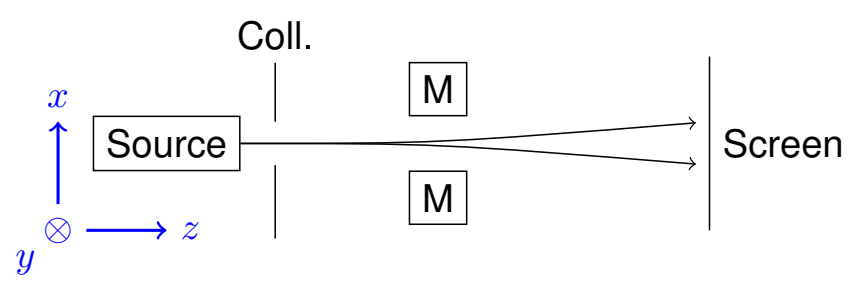

Fig. 1 Basic geometry of the Stern-Gerlach experiment as simulated in the following. The axes are chosen such that the magnetic field is along $y$, while the magnetic field gradient is along $x$. The beam propagates in the $+z$ direction.

field gradient, which exerts a force on the magnetic moment of the particle, is assumed to be in the $y$-direction (Fig. 1). An explicit definition of the fields is given below; Fig. 2A shows how this magnetic field geometry can be realised by a quadrupole magnet arrangement.

The spin state denoted $|\alpha\rangle$ is defined here as the eigenstate of spin angular momentum in the $y$-direction, with eigenvalue $+\hbar / 2$, and in the opposite sense for the eigenstate $|\beta\rangle$ :

$$
\begin{aligned}
& \hat{S}_{y}|\alpha\rangle=+\frac{1}{2} \hbar|\alpha\rangle \\
& \hat{S}_{y}|\beta\rangle=-\frac{1}{2} \hbar|\beta\rangle
\end{aligned}
$$

where $\hat{S}_{y}$ is the operator for spin angular momentum along the $y$-axis. The magnetic moments of the particles are in the opposite direction as the angular momentum (for negative magnetogyric ratio, as for the electron). The field gradient, which is along the $x$-axis, deflects the particles in the state $|\alpha\rangle$ "upwards" (i.e. in the positive $x$-direction), leading at the exit of the apparatus to a spatial wavefunction localized in the "up" branch of the beam, denoted here $|\uparrow\rangle$. Hence, if the particle 
A
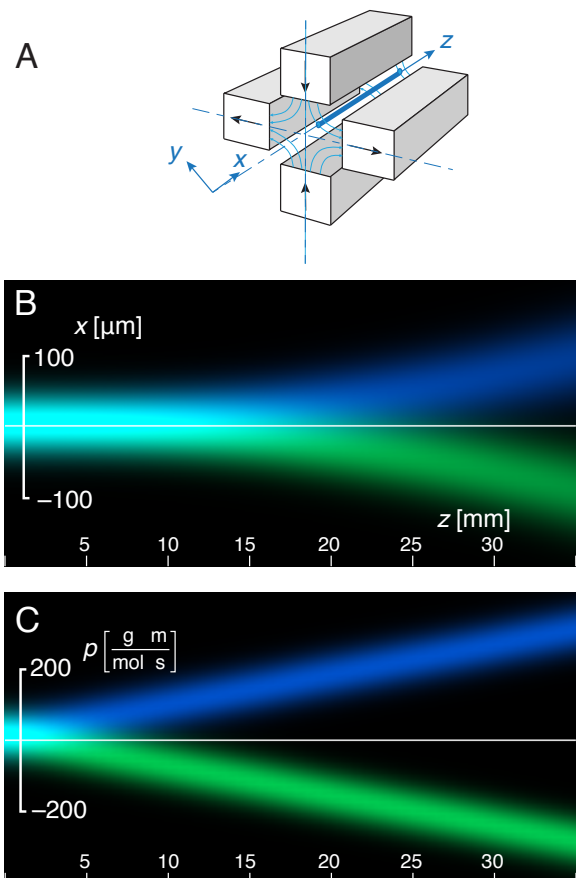

Fig. 2 A: Magnetic field gradient generated by a quadrupole arrangement of permanent magnets. The beam path indicated (solid blue line) passes through a region where the magnetic field is parallel to the $y$-axis, but varies in magnitude along the $x$-axis (uniaxial field gradient). B: Spatial beam trace in the Stern-Gerlach experiment. C: Beam trace in the transverse momentum dimension.

entering the apparatus is initially in spin state $|\alpha\rangle$, it exits the apparatus with a total quantum state given by a direct product of the spatial and spin states, as follows:

$$
\Psi=|\uparrow\rangle \otimes|\alpha\rangle
$$

Similarly, particles in spin state $|\beta\rangle$ are deflected "downwards", leading to a total quantum state as follows:

$$
\Psi=|\downarrow\rangle \otimes|\beta\rangle
$$

More difficulties arise when one considers the behaviour of a particle which is not prepared in an angular momentum eigenstate along the magnetic field, on entering the SG apparatus. For example, consider a particle which enters the SG apparatus in a quantum superposition state of the form

$$
|-z\rangle=2^{-1 / 2}(|\alpha\rangle+|\beta\rangle)
$$

This state is an eigenstate of spin angular momentum along the beam direction, i.e. perpendicular to the magnetic field gradient:

$$
\hat{S}_{z}|-z\rangle=-\frac{1}{2} \hbar|-z\rangle
$$

According to conventional quantum theory, this superposition state evolves in the SG experiment into to a total quantum state of the form

$$
\Psi=2^{-1 / 2}\left(\mathrm{e}^{\mathrm{i} \phi_{\alpha}}|\uparrow\rangle \otimes|\alpha\rangle+\mathrm{e}^{\mathrm{i} \phi_{\beta}}|\downarrow\rangle \otimes|\beta\rangle\right)
$$

where $\phi_{\alpha}$ and $\phi_{\beta}$ are phase factors. An entangled state of this kind cannot be factorized into spin and space components. It is not localized in the "up" or the "down" branches of the beam, but appears in both places at the same time. Only when the particle is observed at the end of the experiment, does the wavefunction "collapse", leading to the particle being localised in either the "up" or "down" branches of the beam, with probabilities given by the square magnitude of the superposition coefficients.

Not surprisingly, the semi-classical approach of WW encounters great difficulties with treating the dynamics of an entangled quantum state such as that given in eq. 6. How does one even write down the force exerted by a potential field on a particle which is in two places at the same time? In attempting a semi-classical description, WW found it necessary to ignore the spatial delocalization of the particle, and to introduce an extraneous fluctuating field, to obtain qualitatively reasonable results. However, there is no experimental evidence for a role for dissipation in the SG experiment, and the conventional quantum theory does not need to invoke such a concept.

By attempting a semi-classical description, Wennerström and Westlund have exposed a curious reticence in the scientific literature to describe in detail what happens in the SG experiment. The outcome is well-known, and is part of current scientific culture. Nevertheless, it is hard to find an explicit description of how the quantum state evolves in space and time, as the particle traverses the SG apparatus, according to conventional quantum mechanics. How does the entangled state in eq. 6 arise, in detail? Apart from the recent attempt by WW, which uses a semi-classical description, we are only aware of one attempt at a detailed description, and that uses the unconventional Bohm description of quantum mechanics $^{4}$. In the discussion below, we use numerical simulations based on an extended Wigner function formulation of conventional Schrödinger quantum mechanics, to help visualise the quantum evolution.

\section{Extended Wigner functions}

The Wigner function formalism ${ }^{5-7}$ provides a compact description of spatial quantum states in terms of a quasi- distribution function in phase space. It incorporates essential features of the spatial quantum state such as its coherence length and the momentum distribution in a natural manner, and provides an intuitive picture of how the position and momentum distributions evolve in time.

Formally, the Wigner function is defined as a Weyl integral transform of the density operator ${ }^{8} \hat{\rho}=|\psi\rangle\langle\psi|$, of the following form:

$$
W(x, p)=\frac{1}{h} \int e^{-\frac{i p s}{\hbar}}\left\langle x+\frac{s}{2}|\hat{\rho}| x-\frac{s}{2}\right\rangle d s .
$$

In its original form, the Wigner function does not take into account the internal degrees of freedom of the particle, such as spin, and hence cannot be applied directly to the Stern-Gerlach experiment. However, extended Wigner function (EWF) formalisms, which include internal degrees of freedom, have 
been proposed ${ }^{9}$. Here we develop the extended Wigner function formalism, and use it to visualise the detailed quantum dynamics taking place in the Stern- Gerlach experiment and its relatives.

Consider a particle with a finite number of internal quantum states. In the discussion below, we refer to these internal states as "spin states", although the same formalism applies to non-spin degrees of freedom, such as quantized rotational and vibrational states. We extend the Wigner function by combining it with the density operator formalism commonly used in the quantum description of magnetic resonance ${ }^{8}$. The definition of the Wigner function is extended by projecting the density operator onto the spin- state specific position state $|x, \eta\rangle$, where $\eta=\alpha, \beta, \ldots$ denotes the spin state. This results in a Wigner probability density matrix $W_{\eta \xi}(x, p)$, whose elements depend parametrically on the positional variables and their associated momenta:

$$
W_{\eta \xi}(x, p)=\frac{1}{h} \int e^{-\frac{i p s}{\hbar}}\left\langle x+\frac{s}{2}, \eta|\hat{\rho}| x-\frac{s}{2}, \xi\right\rangle d s .
$$

For non-relativistic particles, the Hamiltonian may be written as

$$
\hat{H}=\frac{\hat{p}^{2}}{2 m}+U(x, \hat{S}),
$$

where $x$ is the position, and $\hat{p}$ and $\hat{S}$ denote the operators associated with the momentum, and spin degrees of freedom, respectively. It is convenient to consider the contributions of the kinetic and potential energy parts of the Hamiltonian to the time derivative of the Wigner functions separately. It can be shown through integration by parts that the kinetic energy contribution is

$$
\left[\dot{W}_{\eta \xi}(x, p)\right]_{T}=-\frac{p}{m} \frac{\partial}{\partial x} W_{\eta \xi}(x, p),
$$

while the potential energy part of the Hamiltonian contributes as follows:

$$
\begin{aligned}
& {\left[\dot{W}_{\eta \xi}(x, p)\right]_{U}=} \\
& \frac{1}{i \hbar} \sum_{n} \frac{1}{n !}\left(\frac{\hbar}{2 i}\right)^{n} \frac{\partial^{n} W_{\eta \xi}}{\partial p^{n}}\left[(-)^{n} \frac{\partial^{n} U_{\eta \eta}}{\partial x^{n}}-\frac{\partial^{n} U_{\xi \xi}}{\partial x^{n}}\right] .
\end{aligned}
$$

In this expression, the basis of the spin degrees of freedom has been chosen to diagonalise the potential energy: $U_{\eta \xi}(x)=\delta_{\eta \xi}\langle x, \eta|U(x, \hat{S})| x, \xi\rangle$. Obviously, this is only possible if the potential energy operator at different locations commutes: $\left[U\left(x_{1}, \hat{S}\right), U\left(x_{2}, \hat{S}\right)\right]=0 \forall x_{1}, x_{2}$. An expression corresponding to (11) for the general case is given in the supplementary material.

The series (11) converges rapidly if the coherence length $l_{c}$ of the quantum state represented by the Wigner function is short compared to the length scale of variation of $U$. In the momentum dimension, the Wigner function typically has a Gaussian shape of width $\hbar / l_{c}$, and the derivatives $\partial^{n} W_{\eta \eta} / \partial p^{n}$ scale with $\left(l_{c}\right)^{n}$. By contrast, the spatial derivatives of a harmonic potential with period $L$ scale with $L^{-n}$. Together, the terms in (11) therefore scale as $\left(l_{c} / L\right)^{n} / n$ !. If $l_{c} \ll L$, (11) may be truncated to first order, yielding

$$
\left[\dot{W}_{\eta \xi}(x, p)\right]_{U}=W_{\eta \xi} \frac{U_{\eta \eta}-U_{\xi \xi}}{i \hbar}-\frac{\partial W_{\eta \xi}}{\partial p} \frac{F_{\eta \eta}+F_{\xi \xi}}{2}
$$

where $F_{\eta \eta}(x)=-\partial U_{\eta \eta} / \partial x$ is the force acting on the quantum state $\eta$.

This set of partial differential equations may be integrated numerically, forming the basis of detailed simulations of the quantum state propagation in the presence of inhomogeneous fields. In the form given above, which assumes a diagonal Hamiltonian, the different elements of the Wigner matrix $\left(W_{\eta \xi}\right)$ are decoupled, and therefore evolve independently from each other. If the Hamiltonians in different positions do not commute, however, the full version of (11) applies, which couples the internal states.

We now use these equations to simulate the quantum dynamics during the Stern-Gerlach and Rabi experiments on atomic and molecular beams.

\section{Analysis of the Stern-Gerlach Experiment}

In the Stern-Gerlach experiment, a beam of spin- $1 / 2$ particles is exposed to a lateral magnetic field gradient. We define the axis of the molecular beam apparatus as $z$, and assume that the magnetic field varies in the transverse $x$-direction. The potential energy part of the Hamiltonian in the presence of an external magnetic field $\mathbf{B}$ is then given by

$$
U(\hat{\mathbf{S}}, x)=-\hbar \gamma \mathbf{B}(x) \cdot \hat{\mathbf{S}}
$$

The original magnet design used by Stern and Gerlach ${ }^{10}$ produces divergent magnetic field lines at the location of the beam. This corresponds to a biaxial magnetic field gradient tensor, requiring two spatial dimensions to be included in the Wigner function. To avoid this complication, we use a different arrangement, in which the magnetic field gradient is uniaxial. In this case, the magnetic field lines are all parallel, but vary in density in the direction perpendicular to the magnetic field itself. Magnetic fields of this type occur in quadrupole polarisers, as shown in Fig. 2A.

We assume the magnetic field points along the $y$-axis, and varies linearly in magnitude along the $x$-axis, $\mathbf{B}(x, y, z)=$ $\left(B_{y 0}+x G_{x y}\right) \mathbf{e}_{y}$, where $B_{y 0}$ is the magnetic field at $x=0$, and $G_{x y}=\partial B_{y} / \partial x$. This field is fully consistent with Maxwell's equations, since it satisfies $\nabla \cdot \mathbf{B}=0$. The field gradient has only a single non- zero cartesian component $\nabla \mathbf{B}=G_{x y} \mathbf{e}_{x} \mathbf{e}_{y}$. We choose the spin states $|\alpha\rangle$ and $|\beta\rangle$ as the eigenstates of $\hat{S}_{y}$, such that the matrix elements of the potential part of the Hamiltonian are

$$
\begin{array}{ll}
U_{\alpha \alpha}(x)=-\frac{1}{2} \gamma \hbar B_{y}(x) & U_{\alpha \beta}(x)=0 \\
U_{\beta \alpha}(x)=0 & U_{\beta \beta}(x)=+\frac{1}{2} \gamma \hbar B_{y}(x) .
\end{array}
$$

The resulting equations of motion for the EWF matrix elements are given in the SI. 

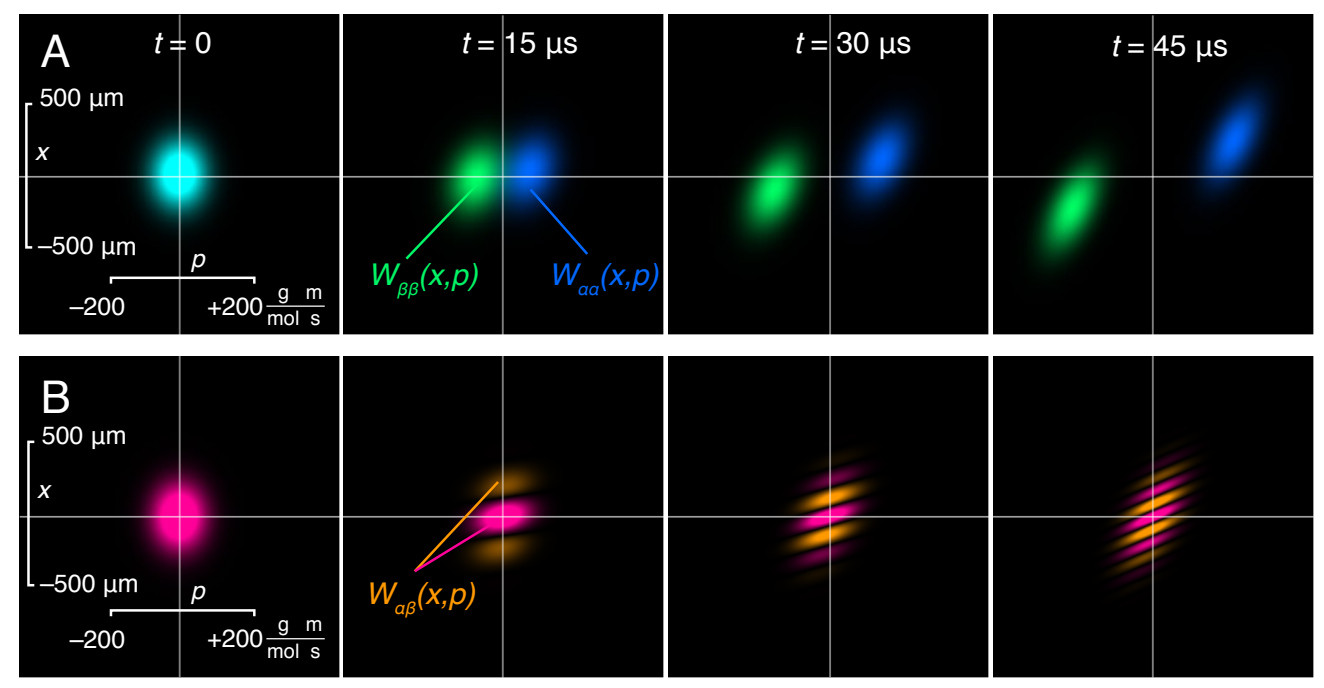

Fig. 3 A: Evolution of $W_{\alpha \alpha}$ and $W_{\beta \beta}$ under the influence of a magnetic field gradient in a Stern-Gerlach experiment on $A g$ atoms in a field gradient of $10 \mathrm{G} \mu \mathrm{m}^{-1}$, moving at a velocity of $550 \mathrm{~m} / \mathrm{s}$ (rms velocity at an oven temperature of $1300 \mathrm{~K}$ ). B: Evolution of the real part of the off-diagonal element $W_{\alpha \beta}$, assuming a coherent state initially polarised along the $x$-axis. The strength of the magnetic field gradient has been reduced by a factor of $5 \times 10^{4}$ compared to $\mathrm{A}$ in order to make the spatial modulation visible.

In its original form, the Stern-Gerlach experiment was conducted on a beam of $\mathrm{Ag}$ atoms emanating from an oven at a temperature of about $1300 \mathrm{~K}$. The magnetic field gradient was of the order of $10 \mathrm{G} / \mathrm{cm}$ over a length of $3.5 \mathrm{~cm}^{1}$. At magnetic fields larger than the hyperfine splitting (about $610 \mathrm{G}^{11}$ in the case of $\mathrm{Ag}$ ), the electron spin of the $\mathrm{Ag}$ atoms is decoupled from the nuclear spin of $\mathrm{Ag}$, and the nuclear spin may be ignored. In this regime the silver atoms may be treated as (electron) spin $1 / 2$ particles. The root mean square velocity of $\mathrm{Ag}$ atoms at $1300 \mathrm{~K}$ is approximately $550 \mathrm{~m} / \mathrm{s}$. After leaving the oven, the $\mathrm{Ag}$ atoms are collimated by a pair of collimation slits $30 \mu \mathrm{m}$ wide and separated by $3 \mathrm{~cm}$. The longitudinal momentum of the silver atoms is approximately $6 \times 10^{4} \mathrm{~g} \mathrm{~mol}^{-1} \mathrm{~ms}^{-1}$. The collimation aspect ratio of 1:1000 therefore results in a transverse momentum uncertainty of $\Delta p=60 \mathrm{~g} \mathrm{~mol}^{-1} \mathrm{~ms}^{-1}$, which corresponds to a $30 \mu \mathrm{m}$ wide beam with a transverse coherence length of about $l_{c}=h / \Delta p \approx 7 \mathrm{~nm}$.

An unpolarised beam entering the magnetic field gradient is represented by a unity spin density matrix, such that $W_{\alpha \alpha}(t=0)=W_{\beta \beta}(t=0)=W_{0}(x, p)$, where the initial state $W_{0}(x, p)$ is a two-dimensional normalised Gaussian function centred at $(x, p)=(0,0)$, with widths given by coherence length $l_{c}$ and the beam width $\Delta x$. The off-diagonal Wigner functions vanish: $W_{\alpha \beta}=W_{\beta \alpha} \equiv 0$, and the diagonal ones can be obtained in closed form by integrating the equations of motion (cf. Methods section).

Fig. 2B shows the projections of the Wigner matrix elements $W_{\alpha \alpha}$ and $W_{\beta \beta}$ onto the spatial axis as a function of position along the beam path in blue and green, respectively. The initially unpolarised beam begins to split after about $10 \mathrm{~mm}$, and is completely separated after $25 \mathrm{~mm}$. As expected, the separation of the two beams grows quadratically along the beam path. The corresponding projection onto the momentum dimension is shown in Fig. 2C. Due to the constant, equal and opposite forces experienced by the two polarisation states, the transverse momentum grows linearly along the beam path. It is interesting to note that in the momentum dimension, the beam is fully polarised beyond $5 \mathrm{~mm}$, while spatial separation does not occur until $25 \mathrm{~mm}$. This is also reflected in the Wigner function "snapshots" shown in Fig. 3A. In these panels, the transverse momentum and position are plotted on the horizontal and vertical axes, respectively. The beam is initially unpolarised and centred. Under the influence of the field gradient, it splits into two separate spots in the momentum direction first, which gradually drift apart in the position dimension, as well. The peaks of the two distributions $W_{\alpha \alpha}$ and $W_{\beta \beta}$ describe parabolic trajectories in the $x, p$-plane in opposite directions. The evolution of the Wigner matrix elements also shows the gradual shearing due to ballistic drift, which leads to divergence of the beams. The final separation of the beams at $z=3.5 \mathrm{~cm}$ amounts to about $200 \mu \mathrm{m}$, which is in quantitative agreement with Stern and Gerlach's observation.

The behaviour of the diagonal Wigner function elements $W_{\alpha \alpha}$ and $W_{\beta \beta}$, shown in Fig. 3A, are readily understood in terms of the magnetic force acting on the spin by the field gradient. The semi-classical analysis developed by $\mathrm{WW}^{3}$ reproduces such behaviour reasonably well. A less intuitive situation is presented by the off-diagonal Wigner function elements $W_{\alpha \beta}$ and $W_{\beta \alpha}$, which correspond to spins in phase-coherent superposition states upon entering the SG apparatus.

Instead of an unpolarised $\mathrm{Ag}$ beam, consider one that has been fully polarised in the $x$ direction before entering the field gradient shown in Fig. 2A. This could be accomplished, for example, by preceding the magnet with a similar one rotated by $90^{\circ}$ about the $z$-axis, and selecting one of the two resulting 

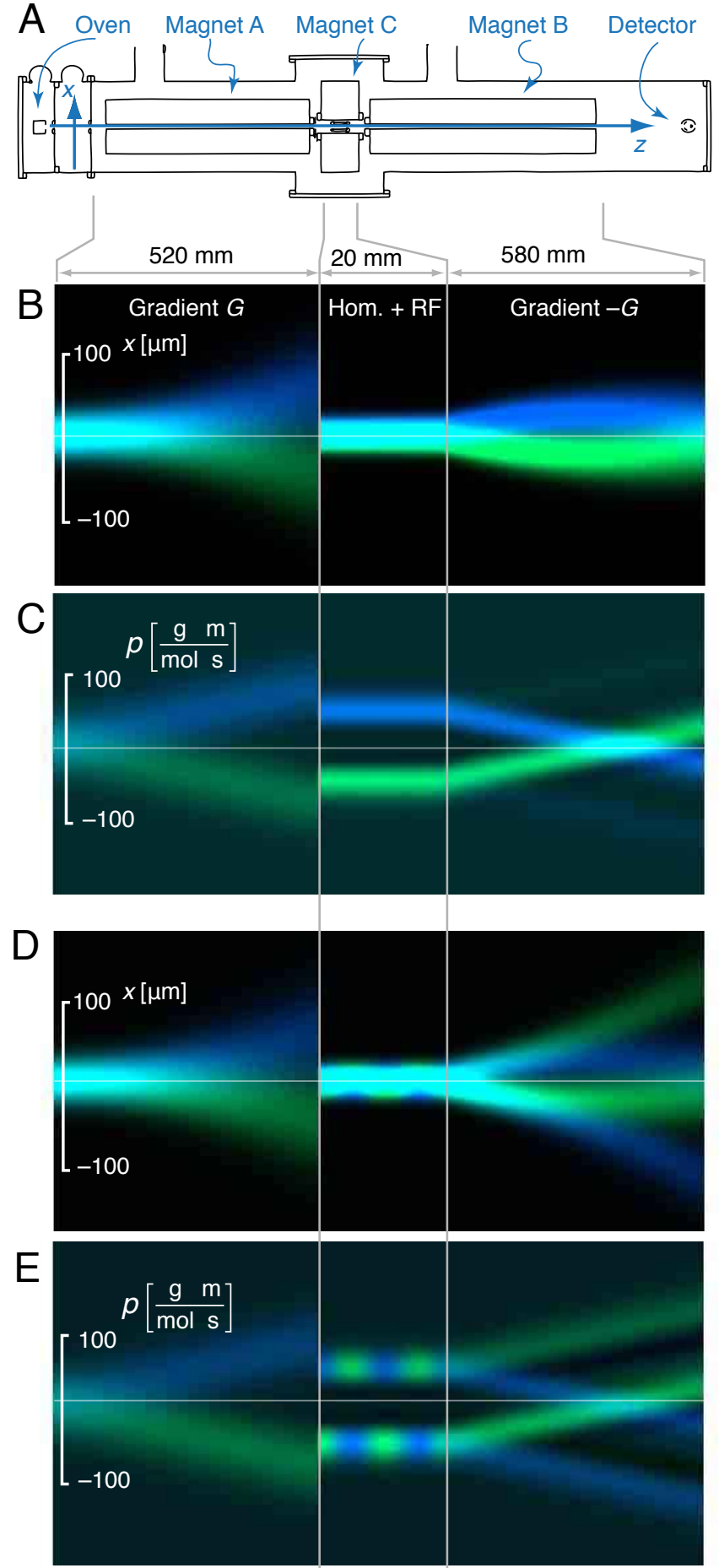

Fig. 4 A: Schematic of the original Rabi apparatus (adapted from $^{12}$ ). B, C: Simulated spatial (B) and momentum (C) beam traces for $B_{y 0}$ far from the resonance condition. D, E Simulated spatial (D) and momentum (E) traces at the magnetic resonance condition. In (E) note the periodic spin state exchanges induced by the resonant radiofrequency field. traces.

Polarisation along the $x$-axis corresponds to a spin quantum state $2^{-1 / 2}(|\alpha\rangle+|\beta\rangle)$, and the initial conditions for the EWF matrix elements are then $W_{\alpha \alpha}=W_{\beta \beta}=W_{\alpha \beta}=W_{\beta \alpha}=$ $\frac{1}{2} W_{0}$. Under the potential energy term (14), the off-diagonal terms undergo a harmonic oscillation with a linearly positiondependent frequency. This leads to a spatial modulation with wave number $k= \pm \gamma G_{x y} t$. At the same time, however, the ballistic drift shears the Wigner function. Therefore, the direction of the phase modulation in the $(x, p)$-plane gradually rotates, and the Wigner function is modulated in both the position and momentum domain. This is shown in Fig. 3B. It should be noted that the spatial frequency of the modulation grows very quickly as a function of time; the field gradient used for the simulation shown in Fig. 3B was reduced by a factor of $5 \times 10^{4}$ compared to Fig. $3 \mathrm{~A}$ in order to make the modulation visible. Under the true field gradient in the $\mathrm{SG}$ experiment $\left(10 \mathrm{G} \mu \mathrm{m}^{-1}\right)$, the wavelength of the spatial modulation after only $45 \mu$ s would already be less than 1 nanometre.

Hence, due to the shear of the EWF due to ballistic drift, the projections of the off-diagonal element of the EWF on either the momentum or the position axis vanish. In the quantum description, no external dissipative agency is required to dephase the off-diagonal elements of the extended Wigner function beyond any hope of recovery: the dephasing happens by itself as a consequence of the quantum dynamics.

\section{Analysis of the Rabi Experiment}

As a second example, we treat the classic magnetic resonance experiment introduced by Rabi and coworkers in order to measure nuclear gyromagnetic ratios ${ }^{12}$. The apparatus is shown in Fig. 4A. It relies on two magnetic field gradients of opposite polarity (Magnets A and B). The first gradient imparts a curvature to the beam path depending on the spin state of the entering particle. This curvature is reversed in the second gradient, thus refocusing the beam. In between the two sets of gradients, there is a region with a homogeneous static magnetic field $B_{y 0}$ (Magnet C), combined with a radio frequency field $B_{x}(t)=B_{1} \cos \left(\omega_{\mathrm{rf}} t\right)$.

The spins undergo nutations at a frequency proportional to $B_{1}$, if $\omega_{\mathrm{rf}}$ is sufficiently close to the Larmor frequency $\omega=-\gamma B_{y 0}$, where $\gamma$ denotes the gyromagnetic ratio. This nutation interferes with the refocusing of the beam, and leads to a measurable decrease in the detected beam intensity. The gyromagnetic ratio can then be inferred from the frequency $\omega_{\text {rf }}$ at which the effect is maximal.

Using the EWF formalism, it is straightforward to simulate this experiment. We assume that the beam consists of $\mathrm{NaF}$ molecules emanating from an oven at $1300 \mathrm{~K}$. The molecules are treated as single spin- $1 / 2$ systems with a gyromagnetic ratio corresponding to ${ }^{19} \mathrm{~F}$; the Na nuclear spin is ignored. The geometry of the apparatus and the magnitudes of the magnetic fields and field gradients have been taken from ref. ${ }^{12}$.

The evolution of the EWF matrix elements has been computed numerically as detailed in the Methods section. Fig. 4B 


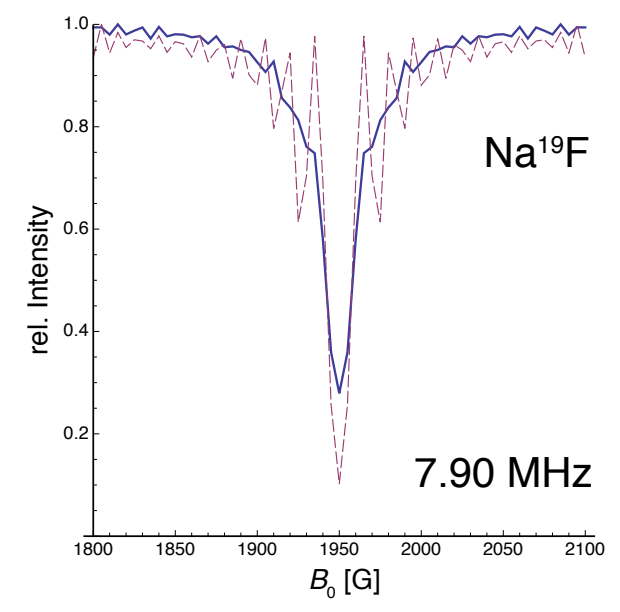

Fig. 5 Predicted beam intensity in the Rabi experiment as a function of magnetic field $B_{0}$ in the homogeneous section. Dashed line: single velocity $v=500 \mathrm{~m} / \mathrm{s}$; solid line: average over 4 different velocities from 450 to $550 \mathrm{~m} / \mathrm{s}$.

and $\mathrm{C}$ show the position and momentum traces in the case of a large resonance offset. The first magnet splits the beam in a manner analogous to the Stern-Gerlach experiment. A narrow collimation slit then admits only the centre of the beam to the homogeneous magnet region. As a result, the beam entering $\mathrm{C}$ is unpolarised in the spatial domain, but completely polarised in the momentum direction (i.e., the transverse momentum and spin states are entangled). The two beams retain their spin "identity", and are then spatially refocused by the inverse field gradient (Magnet B). The situation is different when the magnetic field $B_{y 0}$ is close to resonance (Fig. 4D and E). The spin states are now exchanged periodically under the influence of the resonant radio frequency field in Magnet C. As a result, a large fraction of the beam intensity is further deflected by the refocusing magnet, leading to a decrease of the detector signal.

Fig. 5 shows the computed beam intensity at the detector as a function of $B_{y 0}$, assuming an rf frequency and amplitude of $7.90 \mathrm{MHz}$ and $20 \mathrm{G}$, respectively. Assuming a single velocity of the $\mathrm{NaF}$ molecules leads to a sinc-shaped resonance line (dashed line), which is smoothed out if the results are averaged over a $20 \%$ velocity variation. This results in a line shape that is very similar to the ones reported in the original work by Rabi et $a l^{12}$.

\section{Computational Methods}

In the case of the Stern-Gerlach experiment, the equations of motion are decoupled due to the diagonal potential energy part of the Hamiltonian (14). Moreover, the forces acting on the $\alpha \alpha$ and $\beta \beta$ elements are equal in magnitude and opposite in sign. Under these conditions, the equations of motion can be integrated symbolically. One obtains

$$
\begin{aligned}
& W_{\alpha \alpha}(x, p ; t)=W_{\alpha \alpha}\left(x-\frac{p}{m} t+\frac{F}{2 m} t^{2}, p-F t ; 0\right) \\
& W_{\alpha \beta}(x, p ; t)=\exp \left(-\frac{2 i t}{\hbar} F\left(x-\frac{p t}{2 m}\right)\right) W_{\alpha \beta}\left(x-\frac{p}{m} t, p ; 0\right),
\end{aligned}
$$

where $F=-\partial U_{\alpha \alpha} / \partial x=\frac{1}{2} \gamma \hbar G_{x y}$. Analogous expressions hold for $W_{\beta \beta}$ and $W_{\beta \alpha}$. The results shown above have been obtained by assuming an initially centred Gaussian distribution of width $\Delta p=60 \mathrm{~g} \mathrm{~mol}^{-1} \mathrm{~ms}^{-} 1$ and $\Delta x=30 \mu \mathrm{m}$ at $t=0$. The graphs shown in Fig. 3 were obtained by propagating the initial Gaussian according to (15).

In the case of the Rabi experiment, the potential energy part of the Hamiltonian can no longer be diagonalised in a single coordinate frame throughout the experiment. As a consequence, the equations of motion cannot be solved in closed form. Instead, the extended Wigner functions were represented on a rectangular grid, and the partial derivatives evaluated using a first-order quadrilateral finite element approach. The equations of motion given by (12) were integrated numerically using a fourth-order Runge-Kutta algorithm.

\section{Conclusions}

In summary, we have used an extended Wigner function approach to simulate the quantum dynamics operating in the Stern-Gerlach and Rabi experiments. A detailed picture of the evolution of the quantum state emerges. No dissipative effects are required to reproduce the experimental observations, even when the particle enters the apparatus with a spin polarization perpendicular to the magnetic field gradient.

\section{References}

1 B. Friedrich and D. Herschbach, Physics Today, 2003, 56, 53-59.

2 H. M. França, Foundations of Physics, 2009, 39, 1177-1190.

3 H. Wennerström and P.-O. Westlund, Physical Chemistry Chemical Physics, 2012, 14, 1677-1684.

4 P. R. Holland, The Quantum Theory of Motion, Cambridge University Press, 1993.

5 E. P. Wigner, Phys. Rev., 1932, 40, 749-759.

6 M. Hillery, R. F. O'connell, M. O. Scully and E. P. Wigner, Physics reports, 1984, 106, 121-167.

7 W. B. Case, American Journal of Physics, 2008, 76, 937.

8 U. Fano, Rev. Mod. Phys., 1957, 29, 74-93.

9 A. Arnold and H. Steinrück, Zeitschrift für angewandte Mathematik und Physik ZAMP, 1989, 40, 793-815.

10 W. Gerlach and O. Stern, Zeitschrift für Physik A Hadrons and Nuclei, 1922, 9, 349-352.

11 G. Wessel and H. Lew, Physical Review, 1953, 92, 641.

12 I. Rabi, S. Millman, P. Kusch and J. Zacharias, Phys. Rev., 1939, 55, 526535 . 


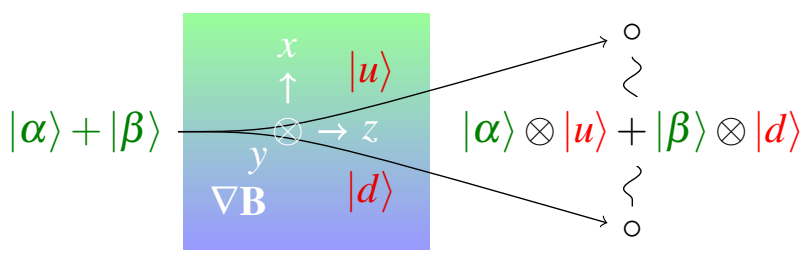

Simulations based on an extended Wigner function formalism allow direct visualisation of the quantum dynamics of the spin/spatial state in Stern-Gerlach and Rabi-type molecular beam experiments. 